\title{
Radio polarization and sub-millimeter observations of the Sombrero galaxy (NGC 4594)^
}

\section{Large-scale magnetic field configuration and dust emission}

\author{
M. Krause ${ }^{1}$, R. Wielebinski ${ }^{1}$, and M. Dumke ${ }^{1,2}$ \\ 1 Max-Planck-Institut für Radioastronomie, Auf dem Hügel 69, 53121 Bonn, Germany \\ e-mail: mkrause@mpifr-bonn.mpg.de \\ 2 European Southern Observatory, Alonso de Cordova 3107, Casilla 19001, Santiago 19, Chile
}

Received 7 July 2005 / Accepted 14 October 2005

ABSTRACT

We observed the nearby early-type spiral galaxy NGC 4594 (M 104, Sombrero galaxy) with the Very Large Array at 4.86 GHz, with the Effelsberg 100-m telescope at $8.35 \mathrm{GHz}$ as well as with the Heinrich Hertz Telescope at $345 \mathrm{GHz}$ in radio continuum. The 4.86 and $8.35 \mathrm{GHz}$ data contain polarization information and hence information about the magnetic fields: we detected a large-scale magnetic field which is to our knowledge the first detection of a large-scale magnetic field in an Sa galaxy in the radio range. The magnetic field orientation in M 104 is predominantly parallel to the disk but has also vertical components at larger $z$-distances from the disk. This field configuration is typical for normal edge-on spiral galaxies. The $345 \mathrm{GHz}$ data pertain to the cold dust content of the galaxy. Despite the optical appearance of the object with the huge dust lane, its dust content is smaller than that of more late-type spirals.

Key words. galaxies: individual: NGC 4594 - galaxies: spiral - galaxies: structure - radio continuum: galaxies - polarization galaxies: magnetic fields

\section{Introduction}

The early-type spiral galaxy NGC 4594 has a spectacular appearance in the optical range with a huge bulge and a prominent dust lane. It is classified as Sa galaxy at a distance of $D=8.9 \mathrm{Mpc}$ (i.e. $10^{\prime \prime}$ are $0.43 \mathrm{kpc}$ ) (Ford et al. 1996). A conspicuous dust lane is obvious in all colour photographs. The dust is distributed in a ring (possibly in two rings) with a radius $\approx 170^{\prime \prime}$ (7.3 kpc) (e.g. Dettmar 1986; Wainscoat et al. 1990).

The rotation curve of the galaxy shows a very steep rise in the central part, with a first maximum at a radius of $10^{\prime \prime}$ $(430 \mathrm{pc})$, then again rising to an unusually high rotation velocity of about $350 \mathrm{~km} \mathrm{~s}^{-1}$ up to $r=8 \mathrm{kpc}$ (Rubin et al. 1985; Wagner et al. 1989). H I line observations of Bajaja et al. (1984) showed two ring/spiral arm structures at $\pm 140^{\prime \prime}(6 \mathrm{kpc})$ and $\pm 170^{\prime \prime}$ from the nucleus. Emission from molecular CO gas was detected only in the inner ring, at a distance of $\pm 140^{\prime \prime}$ from the nucleus (Bajaja et al. 1991).

However, there are numerous observations that suggest that NGC 4594 is a "normal" spiral galaxy: the stellar disk resembles a typical spiral galaxy (Burkhead 1986) which was

* Based on observations with the 100-m telescope of the MPIfR (Max-Planck-Institut für Radioastronomie) at Effelsberg. modelled by a two-armed spiral pattern (van der Burg \& Shane 1986). Recently, the thin spiral disk has been directly revealed by the HST (Hubble Heritage Project, Christian et al. 2003).

Optical studies and a spatial photometric model by Emsellem (1995) suggested that not only extinction but also light scattering by dust is important in NGC 4594. They concluded that the galaxy would even appear "dust free" if it had been viewed face-on. They further estimated the dust content and concluded that significant cold dust should be present in NGC 4594 which should be detectable in the $\mathrm{mm} / \mathrm{sub}-\mathrm{mm}$ wavelength range.

Radio continuum observations of NGC 4594 have at first shown only the strong (and variable) central source (e.g. de Bruyn et al. 1976). With improved dynamic range the disk emission of NGC 4594 was detected (Bajaja et al. 1988). The optical polarization observations of Scarrott et al. (1977) suggested the existence of large-scale magnetic fields, but these were so far not detected in the radio frequency range.

In view of the contradictory publications about the nature of NGC 4594 we decided to make new observations. In this paper we present VLA observations at $4.86 \mathrm{GHz}$ and observations with the Effelsberg 100-m telescope at $8.35 \mathrm{GHz}$, both in total intensity and in linear polarization. As a result we can trace the large-scale magnetic field in NGC 4594. 
Table 1. Source parameters of NGC 4594 and VLA observation details.

\begin{tabular}{|c|c|c|}
\hline \multicolumn{3}{|l|}{ Field centre } \\
\hline B1950 & $\begin{array}{l}\alpha_{50}=12^{\mathrm{h}} 37^{\mathrm{m}} 23^{\mathrm{s}} .4 \\
\delta_{50}=-11^{\circ} 20^{\prime} 55^{\prime \prime}\end{array}$ & \\
\hline $\mathrm{J} 2000$ & $\begin{array}{l}\alpha_{2000}=12^{\mathrm{h}} 39^{\mathrm{m}} 59^{\mathrm{s}} \cdot 4 \\
\delta_{2000}=-11^{\circ} 37^{\prime} 23^{\prime \prime}\end{array}$ & \\
\hline Observing period & $\begin{array}{l}\text { April 3, } 1995 \quad 8 \mathrm{~h} \\
\text { April 30,1995 } 8 \mathrm{~h}\end{array}$ & \\
\hline Frequency & $4.8351 \mathrm{GHz} \quad 4.8851 \mathrm{GHz}$ & \\
\hline Bandwidth & $2 \times 50 \mathrm{MHz}$ & \\
\hline Shortest spacing & $73 \mathrm{~m}$ & \\
\hline Longest spacing & $3.4 \mathrm{~km}$ & \\
\hline Synthesized beam & $23^{\prime \prime}$ & $84^{\prime \prime}$ \\
\hline rms noise (I) $[\mu \mathrm{Jy} / \mathrm{b} . \mathrm{a}]$. & 10 & 25 \\
\hline rms noise (PI) [ $\mu \mathrm{Jy} /$ b.a. $]$ & 7 & 17 \\
\hline
\end{tabular}

We also observed NGC 4594 in the submm range with the HHT at $345 \mathrm{GHz}$ in order get information about the cold dust component and compare this with the model of Emsellem (1995).

The observations and data reduction are described in Sect. 2 and the observational results are presented in Sect. 3. In Sect. 4 we discuss the disk thickness, the magnetic field, the cold dust and the star formation rates and efficiencies. A summary is given in Sect. 5.

For our analysis we have adopted the commonly assumed values for the inclination of the disk as $84^{\circ}$ and the position angle of the major axis as $90^{\circ}$. All values taken from the literature are scaled to the "new" distance of $D=8.9 \mathrm{Mpc}$ for NGC 4594.

\section{Observations and data reduction}

\subsection{Observations with the VLA}

NGC 4594 has been observed in total power and linear polarization at $\lambda 6.2 \mathrm{~cm}$ with the Very Large Array (VLA) ${ }^{1}$ of the National Radio Astronomy Observatory in its D-configuration. Relevant observational parameters are summarized in Table 1. We used 3C 286 and 3C 138 as flux calibrators for the total intensity and for the calibration of the polarization angle. 1243-072 was used as phase calibrator.

The calibration and data reduction were performed with the standard AIPS package at the MPIfR. The data were collected in 2 different periods of 8 hours each in April 1995 with 27 days between the two periods. The calibration and the correction for the antenna polarizations had to be done separately for both observing periods. The UV data were self-calibrated separately in phase and amplitude for both observing periods before they were combined. These combined data were again self-calibrated in phase and amplitude resulting in maps with the natural weighting function. The resulting maps have synthesized beams with $23^{\prime \prime}$ HPBW. The rms noise levels for this

\footnotetext{
1 The VLA is a facility of the National Radio Astronomy Observatory. The NRAO is operated by Associated Universities, Inc., under contract with the National Science Foundation.
}

angular resolution and also for maps smoothed to $30^{\prime \prime}$ and $84^{\prime \prime}$ HPBW are given in Table 1.

\subsection{Observations with the Effelsberg 100-m telescope}

The Effelsberg $\lambda 3.6 \mathrm{~cm}(8.35 \mathrm{GHz})$ observations were made with a receiver in the secondary focus of the 100-m telescope. The single-beam receiver has two channels (RHC, LHC) with total-power amplifiers and an IF polarimeter. The bandwidth is $1.1 \mathrm{GHz}$, the system noise temperature about $25 \mathrm{~K}$ and the resolution is 83". 6 .

We obtained 30 coverages in total of NGC 4594 in February and July 2003. Each coverage has a map size of $20^{\prime} \times 15^{\prime}$ and was scanned along (resp. perpendicular) to the major axis of the galaxy with a scanning velocity of $30^{\prime \prime} / \mathrm{s}$ and a grid size of $30^{\prime \prime}$. For pointing and focussing we observed regularly the source $3 \mathrm{C} 286$. After the observations the pointing was checked again on the strong central radio source of NGC 4594 and corrected if necessary. The flux calibration was also done with $3 \mathrm{C} 286 \mathrm{ac}-$ cording to the flux values of Baars et al. (1977). All coverages were combined (Emerson \& Graeve 1988). The total power map was cleaned for the side lobes of the dirty beam of the strong central source. This was not necessary for linear polarization. The central source itself is not polarized. Map features smaller than the telescope beam appearing in the final maps were filtered out using a Fourier filter technique. The final maps were slightly smoothed to an angular resolution of $84^{\prime \prime} \mathrm{HPBW}$ leading to an rms noise level of the final maps of $650 \mu \mathrm{Jy} /$ beam in total power and $95 \mu \mathrm{Jy} /$ beam in linear polarization.

\subsection{Observations with the HHT}

We observed NGC 4594 at $\lambda 870 \mu \mathrm{m}$ with the Heinrich-HertzTelescope (HHT) ${ }^{2}$ (Baars et al. 1999) on Mt. Graham, Arizona with the 19-channel bolometer array developed by E. Kreysa of the MPIfR. The HPBW of the telescope is $\simeq 23^{\prime \prime}$ at this frequency. The central frequency of the bolometer is about $345 \mathrm{GHz}$ with the highest sensitivity at about $340 \mathrm{GHz}$. The instrument is sensitive between 310 and $380 \mathrm{GHz}$.

The observations were made in March 2000 under varying weather conditions. To calculate the atmospheric opacity we made sky dips regularly. For flux calibration purposes we used the planet Saturn, observed regularly during our observing sessions.

All maps were observed in the Azimuth-Elevation system, by scanning in azimuth and performing data acquisition every $0.5 \mathrm{~s}$. During the observations, the subreflector was wobbled at $2 \mathrm{~Hz}$ in azimuth with a beam throw between $60^{\prime \prime}$ and $120^{\prime \prime}$. In total we observed 22 coverages of NGC 4594, centered on two different positions located $60^{\prime \prime}$ east and west of the nucleus, respectively. The field size of each individual coverage was $450^{\prime \prime} \times 320^{\prime \prime}$.

The data reduction of these observations was performed with the NIC program of the GILDAS software package.

\footnotetext{
2 The HHT was at that time operated by the Submillimeter Telescope Observatory on behalf of Steward Observatory and the MPI für Radioastronomie.
} 


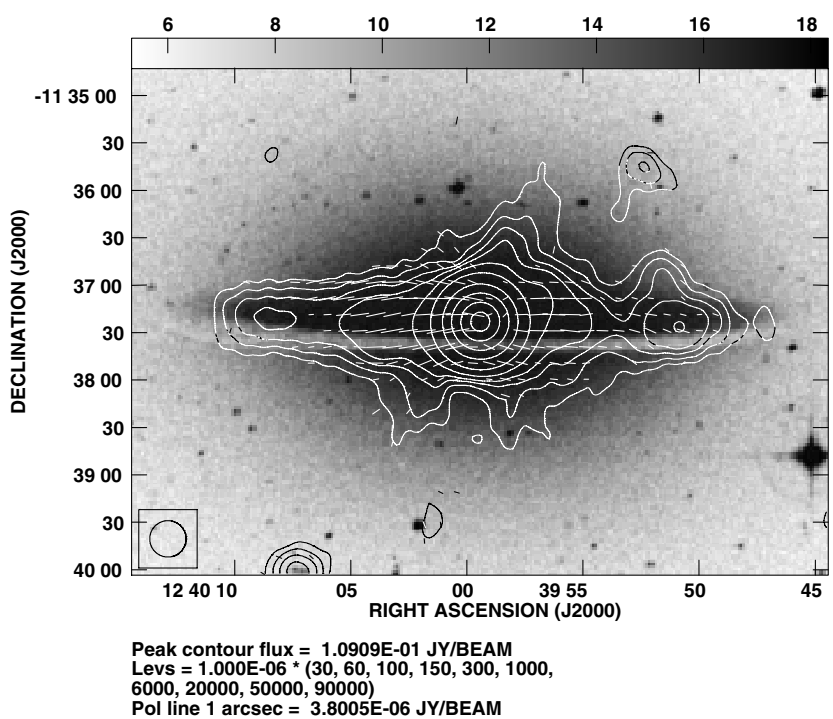

Fig. 1. Full resolution $\left(\right.$ HPBW $\left.23^{\prime \prime} \times 23^{\prime \prime}\right)$ total intensity map of NGC 4594 at $\lambda 6.2 \mathrm{~cm}$ superimposed on the optical photograph of the DSS. The contours are $0.03,0.06,0.1,0.15,0.3,1,6,20,50$, and $90 \mathrm{mJy} / \mathrm{beam}$. The rms noise in the map is $10 \mu \mathrm{Jy} / \mathrm{beam}$. The orientation of the "vectors" gives the observed electric field rotated by $90^{\circ}$, their length is proportional to the observed linearly polarized intensity. The half-power beamwidth is indicated in the bottom left corner.

Additional information about HHT observations and the reduction can be found in Dumke et al. (2004). After baseline subtraction and the elimination of spikes in each single bolometer channel, the atmospheric noise, which is correlated between the 19 channels, was subtracted. The maps were gridded, restored, converted into the RA-Dec system and finally combined with an appropriate weighting. The zero-level of the resulting map was checked and carefully adjusted. Map features smaller than the telescope beam appearing in the final maps were again filtered out using a Fourier filter technique and the map was slightly smoothed to $24^{\prime \prime}$ HPBW. The rms noise level of the final map is about $45 \mathrm{mJy} /$ beam.

\section{Results}

The total intensity map at $\lambda 6.2 \mathrm{~cm}$ in the full resolution (HPBW $23^{\prime \prime} \times 23^{\prime \prime}$ ) including the central source is shown in Fig. 1. The map is superimposed on an optical photograph (DSS) of the galaxy. We see clearly several spur-like features in addition to the nuclear source and the extended disk emission. The agreement with the $\lambda 20 \mathrm{~cm}$ map of Bajaja et al. (1988) is good. We will discuss this aspect in Sect. 3.2.

The total intensity map at $\lambda 3.6 \mathrm{~cm}$ is shown in Fig. 2, also with the DSS as grey plot. The map has an angular resolution of $84^{\prime \prime}$. Again, we see the strong central source with some emission of the extended disk.

\subsection{The central radio source}

The nucleus of NGC 4594 was identified as a LINER by Heckman (1980). Inside the central 15" Burkhead (1986) detected an "inner disk". First evidence for a central black whole

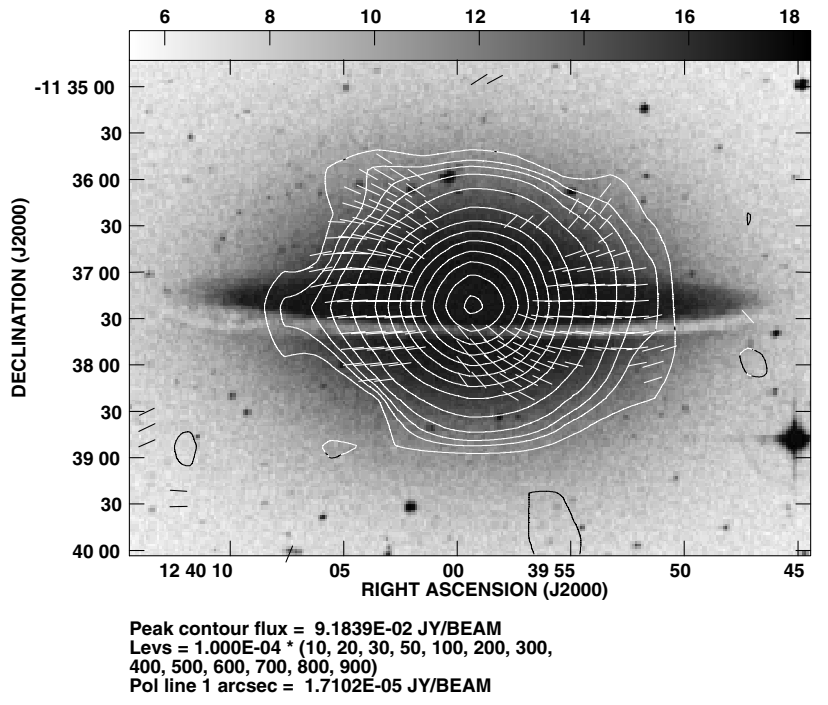

Fig. 2. Total intensity map of NGC 4594 at $\lambda 3.6 \mathrm{~cm}$ observed with the 100-m Effelsberg telescope, superimposed on the optical photograph of the DSS. The contours are 1, 2, 3, 5, 10, 20,..80, $90 \mathrm{mJy} /$ beam. The rms noise in the map is $0.5 \mathrm{mJy} / \mathrm{beam}$. The orientation of the "vectors" gives the observed electric field rotated by $90^{\circ}$, their length is proportional to the observed linearly polarized intensity. The halfpower beamwidth is $84^{\prime \prime} \times 84^{\prime \prime}$.

with a mass $\sim 10^{9} M_{\odot}$ was given by Kormendy (1988) which could later be confirmed by HST observations (Kormendy et al. 1996).

With our angular resolution between 23" and 84" HPBW (corresponding to $1.0-3.6 \mathrm{kpc}$ ) we cannot resolve the nucleus and its surroundings. Hence, we can only give flux densities for the central region at our observed frequencies.

We fitted a Gaussian to the central source at $\lambda 6.2 \mathrm{~cm}$ and find at our epoch a flux density of $S_{6 \mathrm{~cm}}=109 \pm 5 \mathrm{mJy}$. Our present value lies within the range of values compiled by de Bruyn et al. (1976). We could not observe any significant variations of the relative flux density $(\simeq 1 \mathrm{mJy})$ between the two observing periods that were separated by 27 days.

Bajaja et al. (1988) collected nuclear flux density values at $\lambda 20 \mathrm{~cm}$ for the period 1971-1986. At this wavelength the nuclear flux density remained rather constant between 1971 and 1980 while it increased in the following 4 years by about $67 \%$. The flux density dropped after 1985 . Unfortunately, we could not find recent flux density values at $\lambda 6.2 \mathrm{~cm}$ in the literature to follow the temporal evolution in detail.

A Gaussian fit to the central source at $\lambda 3.6 \mathrm{~cm}$ gives a flux density of $S_{3.6 \mathrm{~cm}}=90 \pm 10 \mathrm{mJy}$.

We also fitted a Gaussian to the central source at $\lambda 870 \mu \mathrm{m}$ (Fig. 7, described in Sect. 3.4) and got a flux density of $S_{870 \mu \mathrm{m}}=230 \pm 35 \mathrm{mJy}$.

\subsection{The extended continuum emission}

We subtracted the central source by fitting a Gaussian to the peak emission from the map in Fig. 1. This allows us to study the extended disk component as shown in Fig. 3. The extended disk emission shows four maxima along the major axis and 


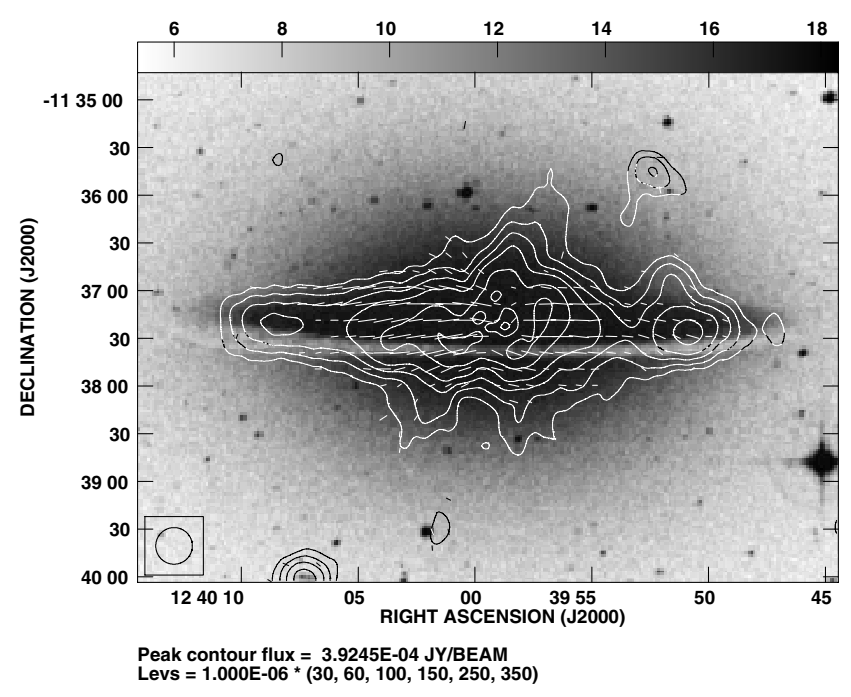

Fig. 3. Total intensity map of the disk emission of NGC 4594 (the central source has been subtracted) at full resolution (HPBW $23^{\prime \prime} \times$ $\left.23^{\prime \prime}\right)$ at $\lambda 6.2 \mathrm{~cm}$ superimposed on the optical photograph of the DSS. The contours are 30,60,100, 150, 250, and $350 \mu \mathrm{Jy} / \mathrm{beam}$. Further notation is as in Fig. 1.

several spurs at high $z$-values. The two outer, most prominent peaks along the major axis are located at a distance of about $130^{\prime \prime}$ east and $120^{\prime \prime}$ west of the nucleus. This is near the position of the inner HI "ring" ( $r=144$ ") as found by Bajaja et al. (1984) and the location of detected molecular gas in NGC 4594 ( $r=140^{\prime \prime}$, Bajaja et al. 1991). The peaks at $\lambda 6.2 \mathrm{~cm}$ are further in the region of the dust rings and coincide with the peaks in the radio continuum observations at $\lambda 20 \mathrm{~cm}$ (Bajaja et al. 1988). Rather than a ring, they may outline the tangential directions of spiral arms in NGC 4594.

The two additional maxima in the central disk area can best be investigated by studying the intensity distribution along the major axis. These cuts were made for both, $\lambda 6.2 \mathrm{~cm}$ and $\lambda 20 \mathrm{~cm}$, and are shown in Fig. 4. They look very similar with only some small differences between the two cuts. The inner maxima at $\Delta \alpha= \pm 25^{\prime \prime}$ are nearly equally high, indicating the same spectral index. The eastern inner maximum has an additional structure along the $\alpha$ axis. Also the maxima at $\Delta \alpha \approx \pm 125^{\prime \prime}$ have a similar ratio east and west of the nucleus, respectively.

Integrating the total intensity (without the central source) in ellipses leads to an integrated flux density of $6.0 \pm 0.5 \mathrm{mJy}$ at $\lambda 6.2 \mathrm{~cm}$. The integrated flux density at $\lambda 20 \mathrm{~cm}$ is found to be $13.4 \mathrm{mJy}$ (Bajaja et al. 1988). Hence, the averaged spectral index for NGC 4594 is $\alpha=0.68$. This value is typical for a synchrotron spectrum (cf. also Sect. 4.2.2). It may, however, still be influenced by missing spacings in both maps and the amount of their effect is difficult to estimate.

The spurs at high $z$-values seen in both, the $\lambda 6.2 \mathrm{~cm}$ map and the $\lambda 20 \mathrm{~cm}$ map (Fig. 1 in Bajaja et al. 1988), are in similar positions, suggesting outflows as a result of central activity of NGC 4594. The spurs at $\lambda 6.2 \mathrm{~cm}$ are more pronounced than at $\lambda 6.2 \mathrm{~cm}$,. This may indicate a thermal origin, suggesting that $\mathrm{H} \alpha$ emission is involved in the outflow.

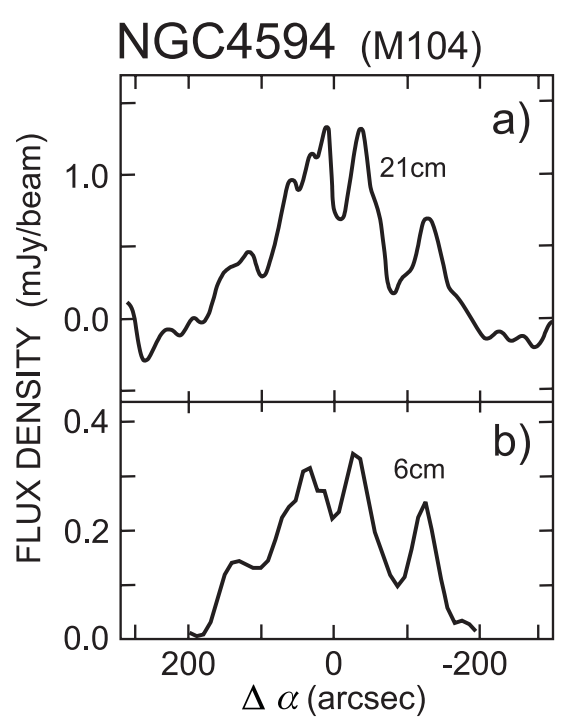

Fig. 4. Brightness profile along the major axis of NGC 4594 of the $\lambda 21 \mathrm{~cm}$ observations of Bajaja et al. (1988) with $30^{\prime \prime} \times 30^{\prime \prime}$ a), and along our map at $\lambda 6.2 \mathrm{~cm}$ smoothed to $\left.30^{\prime \prime} \times 30^{\prime \prime} \mathbf{b}\right)$. The central source was subtracted in both maps before making the profile.

\subsection{The polarized emission}

The $\lambda 6.2 \mathrm{~cm}$ data show considerable linear polarization in the disk (see Fig. 5). In both, the central area and also the outer disk region polarized emission has definitively been detected. Bajaja et al. (1988) suggested a preliminary detection of linear polarization mainly outside the disk along a spur at $\lambda 20 \mathrm{~cm}$ with an orientation of the magnetic field along the spur.

The orientation of the vectors in Figs. 1, 3 and 5 gives the orientation of the observed electric field rotated by $90^{\circ}$, hence roughly the magnetic field orientation (as Faraday rotation is small in NGC 4594 (cf. Sect. 4.2.1)). (Note that the extension of the vectors plotted in the figures is slightly different as we plotted the vectors up to the first contour in each figure which corresponds to about $1.7 \times \sigma(\mathrm{PI})$ in Figs. 1 and 3 but to $3 \times \sigma(\mathrm{PI})$ in Fig. 5 .) The vectors are orientated regularly, mainly parallel to the galactic disk. Deviations from the alignment with the disk are seen mainly above and below the disk (see also Fig. 1) and indicate vertical magnetic field components. The lengths of the vectors are proportional to the degree of linear polarization. It is rather high with values between $20 \%$ and $30 \%$.

The integrated flux density in polarized emission is $0.9 \pm$ $0.1 \mathrm{mJy}$. This leads to an averaged degree of linear polarization of $15 \pm 3 \%$ over the whole galaxy.

The observation at $\lambda 3.6 \mathrm{~cm}$ with the $100-\mathrm{m}$ Effelsberg telescope also clearly shows linearly polarized emission (Figs. 2 and 6). Due to the rather low angular resolution of only $84^{\prime \prime}$ HPBW and the higher frequency we can only detect the main polarized regions. The vector orientation suggests good agreement with those at $\lambda 6.2 \mathrm{~cm}$. The correction of Faraday rotation and the deduced magnetic field orientation will be described in Sect. 4.2.1.

The degree of linear polarization is only around $10 \%$, most probably because of strong beam depolarization due to the large beam size. 


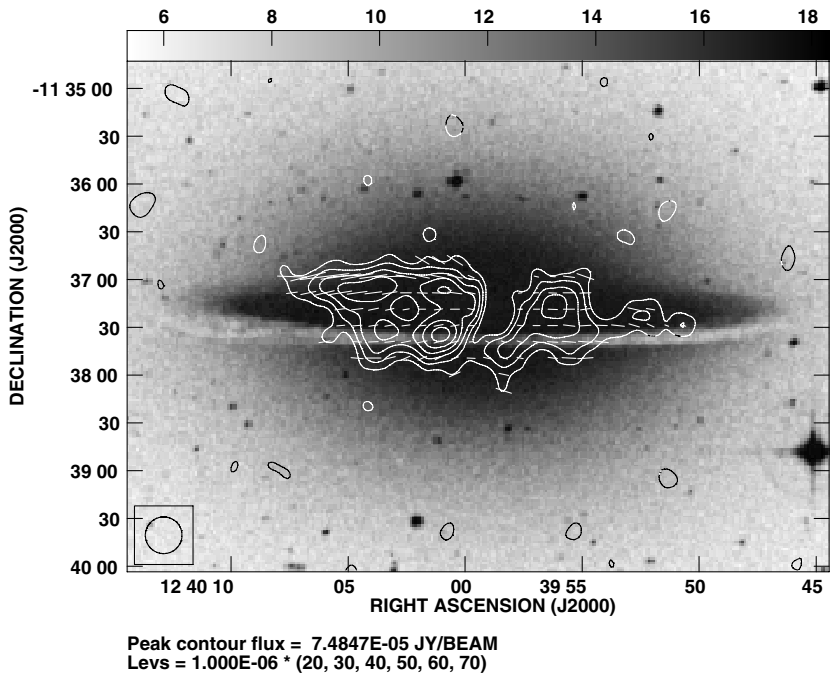

Fig. 5. Full resolution (HPBW $23^{\prime \prime} \times 23^{\prime \prime}$ ) map of the linear polarization of NGC 4594 at $\lambda 6.2 \mathrm{~cm}$ superimposed on the optical photograph of the DSS. The contours are $20,30,40, \ldots \mu \mathrm{Jy} /$ beam. The rms noise in the map is $7 \mu \mathrm{Jy} /$ beam. The orientation of the "vectors" gives the observed electric field rotated by $90^{\circ}$ as in Figs. 1 and 3, (only their extension is slightly smaller as they are plotted up to the first contour of PI). The length of the vectors is proportional to the degree of linear polarization which is between $20 \%$ and $30 \%$ in the region of strong linear polarization. The half-power beamwidth is indicated in the bottom left corner.

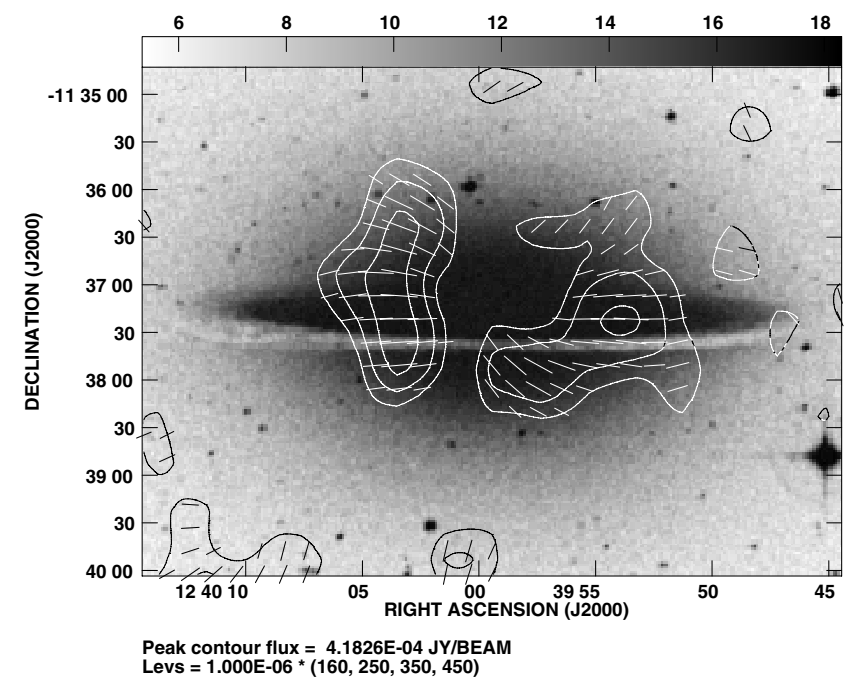

Fig. 6. Linearly polarized intensity of NGC 4594 at $\lambda 3.6 \mathrm{~cm}$ observed with the 100-m Effelsberg telescope, superimposed on the optical photograph of the DSS. The contours contours are 160, 250, 350, and $450 \mu \mathrm{Jy} /$ beam. The rms noise in the map is $95 \mu \mathrm{Jy} /$ beam. The orientation of the "vectors" gives the observed electric field rotated by $90^{\circ}$, their lengths is proportional to the observed linearly polarized intensity. The half-power beamwidth is $84^{\prime \prime} \times 84^{\prime \prime}$.

\subsection{Dust emission}

We have also observed the radio continuum emission of the dust at $\lambda 870 \mu \mathrm{m}$ in NGC 4594 as described in Sect. 2. The final map is shown in Fig. 7. This map has the original resolution of 24" HPBW and shows no significant emission at $\lambda 870 \mu \mathrm{m}$ in the disk of NGC 4594 above the noise level of

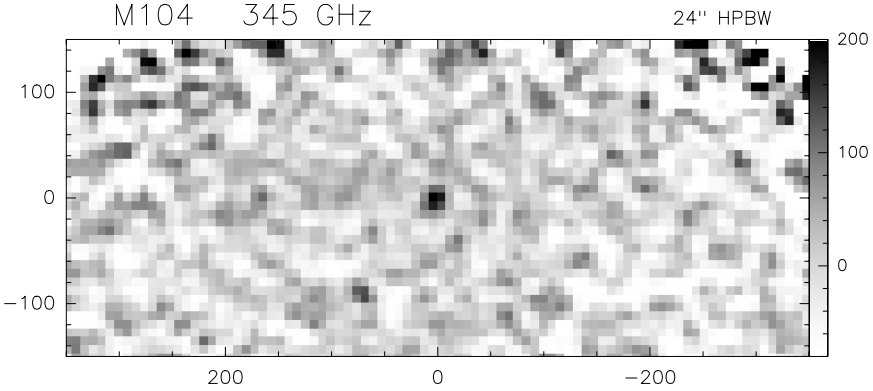

Fig. 7. Radio continuum map of NGC 4594 at $\lambda 870 \mu \mathrm{m}$ observed with the HHT on Mt. Graham. The map is centered on the nucleus of NGC 4594, with relative positions given in arcsec. The greyscale gives the intensity in $\mathrm{mJy} / \mathrm{beam}$. The rms noise in the map is $45 \mathrm{mJy} / \mathrm{beam}$ at a linear resolution of $24^{\prime \prime}$ HPBW.

about $45 \mathrm{mJy} / \mathrm{beam}$. At the upper left and right corner of the map we see an increase of the noise level at the edge of the coverage area. Also smoothing the map to 40" HPBW gives no hint to an extended emission at this wavelength in the disk NGC 4594. However, Fig. 7 clearly reveals the emission of the nuclear region at $\lambda 870 \mu \mathrm{m}$ with $S_{870 \mu \mathrm{m}}=230 \pm 35 \mathrm{mJy}$ as described in Sect. 3.1. Within the central 5" (200pc), a nuclear dust lane with a weak symmetric counterpart was observed by Emsellem (1995). This feature was later interpreted by Emsellem \& Ferruit (2000) as a nuclear bar with a projected length of $>1.5^{\prime \prime}(50 \mathrm{pc})$. The dust lane may be at least partly responsible for our central dust emission which cannot be resolved by our observations.

\section{Discussion}

\subsection{Disk thickness}

In order to determine the thickness of the synchrotron emission at $\lambda 6.2 \mathrm{~cm}$ we examined the distribution of the radio emission perpendicular to the disk plane (i.e. in $z$-direction). We applied a procedure as described by Dumke et al. (1995): we made a strip integration of the total intensity (Fig. 3) in $z$-direction with strips parallel to the major axis east and west of the nucleus and a width of $10^{\prime \prime}$. We varied the length of each strip parallel to the major axis between $50^{\prime \prime}$ and $100^{\prime \prime}$ up to distance of $170^{\prime \prime}$ from the nucleus.

The $z$-distribution of the total intensity of NGC 4594 does not only reflect its disk height but is also affected by inclination effects and the beamwidth of the observations. We corrected for this as described by Dumke et al. (1995) and fitted either a Gaussian or an exponential function to the $z$-distribution, either with one component or two components. Least-square fits show lowest $\chi^{2}$ values for a single component Gaussian distribution.

The resultant scale height for the disk thickness of the $\lambda 6.2 \mathrm{~cm}$ radio emission in NGC 4594 is $33^{\prime \prime} \pm 3^{\prime \prime}(1.4 \pm$ $0.2 \mathrm{kpc}$ ) within $r= \pm 100^{\prime \prime}(4.3 \mathrm{kpc})$ (note that the larger value of $3 \mathrm{kpc}$ given in Krause (2003) refers to the same analysis but assumed a larger distance of NGC 4594). The scale height for the linearly polarized emission has about the same value.

This result differs from the analysis of other spiral galaxies seen edge-on, like NGC 891, NGC 3628, NGC 4565, and 
NGC 5907 (Dumke \& Krause 1998; Dumke et al. 2000). In all those galaxies the $z$-distribution of the radio emission was better described by a two component exponential function with very similar scale heights for all these galaxies of $300 \mathrm{pc}$ for the thin and $1.8 \mathrm{kpc}$ for the thick disk. In contrast to those galaxies, NGC 4594 has a huge bulge with an elliptical mass distribution. The expected $z$-distribution of a relatively thin layer (the disk) inside a nearly spherical gravitational potential is in fact a Gaussian (Combes 1991).

\subsection{Magnetic field}

\subsubsection{Faraday rotation and magnetic field structure}

The observed electric vectors are rotated by the Faraday effect. The amount of Faraday rotation can be determined by calculating the rotation measure RM between different wavelengths. Correction of the observed electric vectors according to these RMs and rotation by $90^{\circ}$ leads to the intrinsic direction of the magnetic field in the sky plane. The RM value itself depends on the strength of the magnetic field component parallel to the line of sight, its sign indicates the direction of this parallel field component.

We determined the RM between $\lambda 3.6 \mathrm{~cm}$ and $\lambda 6.2 \mathrm{~cm}$ at an angular resolution of $84^{\prime \prime} \mathrm{HPBW}$, the resolution of the $\lambda 3.6 \mathrm{~cm}$ observations. We determined the RM only for those points for which the polarized intensity exceeds 2 times the noise value at that wavelength, resp. The calculated RM lies in the range between -200 and $200 \mathrm{rad} / \mathrm{m}^{2}$ with most values between -100 and $100 \mathrm{rad} / \mathrm{m}^{2}$. The $n \pi$ ambiguity (i.e. the RM value that corresponds to a Faraday rotation of $n \cdot 180^{\circ}$ ) between these two wavelengths is as high as $1216 \mathrm{rad} / \mathrm{m}^{2}$ for $n=1$ and hence not relevant. This also implies that NGC 4594 is Faraday thin at both wavelengths. As there are no strange jumps in the RM map and the degree of linear polarization is quite similar at both wavelengths when smoothed to the same resolution of $84^{\prime \prime}$ HPBW, we can assume that the $\lambda^{2}$ dependence of the RM is valid (Sokoloff et al. 1998).

We do not see a systematic change of the RM values above and below the major axis of NGC 4594 that would indicate a systematic change (of the direction) of the line of sight component of the magnetic field above and below the major axis. The RM structure looks quite smooth with both, positive and negative values on both sides of the galaxy along the major axis.

We estimated the galactic foreground rotation measure towards NGC 4594 by averaging the RM values of background sources within a distance of $20^{\circ}$ from NGC 4594 (Simard-Normandin et al. 1981; P. Kronberg, priv. comm.). We omitted two very strongly deviating sources as their RM values may rather reflect their intrinsic Faraday rotation than the galactic RM. The average of the remaining 18 sources is $-4 \pm 15 \mathrm{rad} / \mathrm{m}^{2}$. Also within their errors it infers an additional rotation at $\lambda 6.2 \mathrm{~cm}$ of at most $5^{\circ}$. We conclude that the foreground RM is negligible and that the observed RM is exclusively associated with NGC 4594.

The corresponding corrections for Faraday rotation for most of the observed electric vectors at $\lambda 6.2 \mathrm{~cm}$ is smaller than $\pm 22^{\circ}\left(|\mathrm{RM}| \leq 100 \mathrm{rad} / \mathrm{m}^{2}\right)$. The corrected vectors, rotated

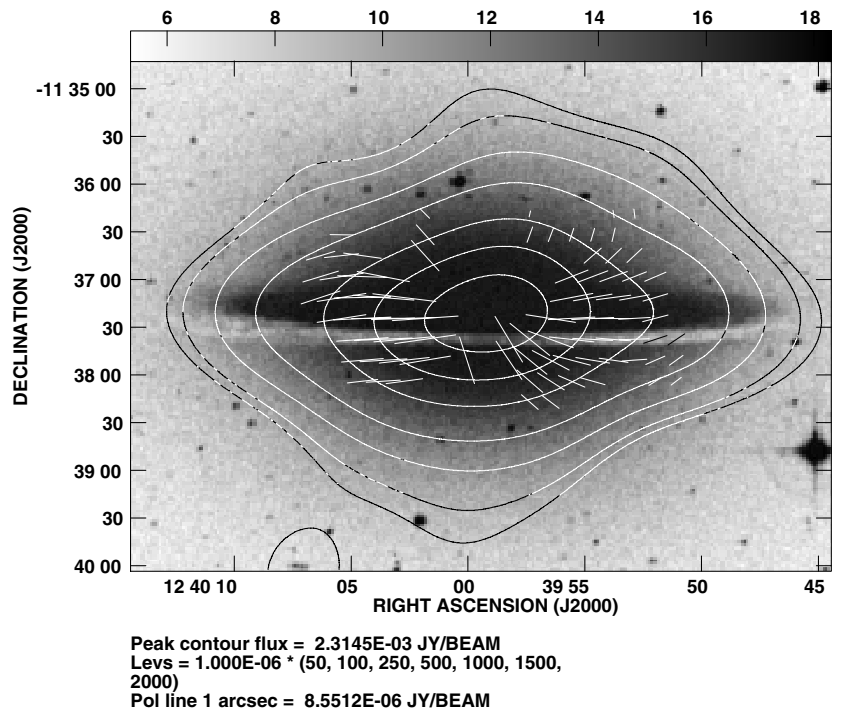

Fig. 8. Smoothed map (HPBW $84^{\prime \prime} \times 84^{\prime \prime}$ ) of the total disk emission of NGC 4594 at $\lambda 6.2 \mathrm{~cm}$ superimposed on the optical photograph of the DSS. The contours are 50,100, 250, 500, 1000, .. $\mu \mathrm{Jy} / \mathrm{beam}$. The orientation of the "vectors" gives the intrinsic magnetic field orientation, their length is proportional to the linearly polarized intensity at $\lambda 6.2 \mathrm{~cm}$.

by $90^{\circ}$, are shown in Fig. 8 superimposed on the smoothed $\lambda 6.2 \mathrm{~cm}$ total intensity map of the disk emission (the central source has been subtracted before smoothing). The vectors give the intrinsic magnetic field orientation in NGC 4594 at the angular resolution of 84" HPBW. Because of the large beam size compared to the angular extent of NGC 4594 and the small signal-to-noise ratio of the polarized emission at $\lambda 3.6 \mathrm{~cm}$, this correction is still quite coarse. Especially the small signal-tonoise ratio at $\lambda 3.6 \mathrm{~cm}$ of $\leq 5$ may lead to an uncertainty on position angle of about $10^{\circ}$ for each single data point. Together with the (smaller) uncertainty at $\lambda 6.2 \mathrm{~cm}$ the error in each single data point in the RM may be even $\leq 100 \mathrm{rad} / \mathrm{m}^{2}$. However, the smooth appearance of the RM-map and the similarity of the observed vectors at $\lambda 3.6 \mathrm{~cm}$ (Fig. 6) with those at the better linear resolution at $\lambda 6.2 \mathrm{~cm}$ (Fig. 5) indicates strongly that the true errors in the RM are much smaller. Further, the signal-tonoise ratio at $\lambda 6.2 \mathrm{~cm}$ is as large as $\leq 10$, inferring an error on position angle of only $\leq 3^{\circ}$.

Hence, from Fig. 8 we can deduce conclusions for the magnetic field structure in NGC 4594 which are in full agreement with the higher resolved map in Fig. 5: along the inner galactic disk the magnetic field orientation is mainly parallel to the disk in the midplane except in the innermost $50^{\prime \prime}$. There and generally at higher $z$-values above and below the disk the magnetic field has also significant vertical components.

\subsubsection{Magnetic field strength}

We estimated the magnetic field strength in the two regions of highest linear polarization east and west of the nucleus at $|r| \simeq 20^{\prime \prime}$ to $110^{\prime \prime}$ from the values of the $\lambda 6.2 \mathrm{~cm}$ map with 23" HPBW angular resolution (see Fig. 5). The estimation has been done under the assumption of energy-density 
equipartition between the energy of the magnetic field and cosmic ray electrons with the revised formula (Beck \& Krause 2005). The revised formula is based on the integration of the energy spectrum of the cosmic-ray protons and not on the integration over the radio frequency spectrum which introduces an implicit dependence on the field strength. We adopt a cosmic ray proton to electron ratio of $K_{0}=100$, as in earlier publications.

The spectral index between $\lambda 6.2 \mathrm{~cm}$ and $\lambda 20 \mathrm{~cm}$ for these two regions was estimated to $\alpha=0.73$ (east) and $\alpha=0.72$ (west) which is very well in agreement with the value averaged for 74 external galaxies (Niklas et al. 1997) and not far away from the estimated integrated spectral index for this galaxy ( $\alpha=0.68$, see Sect. 3.2). Assuming the values of Niklas et al. (1997) for the thermal fraction of $20 \%$ at $\lambda 6.2 \mathrm{~cm}$ and $8 \%$ at $\lambda 20 \mathrm{~cm}$, we obtain a nonthermal spectral index for both regions of about 0.85 . The nonthermal degree of linear polarization is found to be $29 \%$ (east) and $30 \%$ (west). We assumed (I) a mainly toroidal field configuration plane parallel to the disk of the galaxy with an inclination of $84^{\circ}$ (i.e. nearly edge-on) and a line of sight of $9 \mathrm{kpc}$ or (II) strong vertical field components $\left(i=6^{\circ}\right)$ with a line of sight of $4 \mathrm{kpc}$. In both cases the resulting field strengths for both regions are $6 \pm 1 \mu \mathrm{G}$ for the total field and $3 \pm 1 \mu \mathrm{G}$ for the regular magnetic field component with a cosmic energy density $\epsilon_{\mathrm{cr}}=1.2 \pm 0.310^{-12} \mathrm{erg} \mathrm{cm}^{-3}$. The averaged total field strength estimated from the integrated intensity at $\lambda 6.2 \mathrm{~cm}$ averaged over the whole galaxy (without the central source) is $4 \pm 1 \mu \mathrm{G}$ with $\epsilon_{\mathrm{cr}}=0.8 \pm 0.310^{-12} \mathrm{erg} \mathrm{cm}^{-3}$. Hence, the magnetic field strengths in NGC 4594 are in the lower range of those of normal spiral galaxies (e.g. Krause 2003; Beck 2004).

\subsection{Morphology: bulge, bar and magnetic field}

From the Hubble Heritage image of NGC 4594 it can clearly be seen that NGC 4594 is a galaxy with a thin disk that is structured, possibly by spiral arms, and an enormous halo or bulge (Christian et al. 2003). There has been a long and controversial discussion in the literature about the huge spherical bulge in NGC 4594. An appealing explanation is that the spherical bulge is due to a dissolving bar. Such a dynamical interpretation of the ring/spiral structure, a dissolved bar and possibly a bulge formation has first been proposed by Emsellem (1995). It was already known at that time that the colour of the bulge is about similar to that of the disk (Dettmar 1986). Later simulation of galaxy evolutions (e.g. Combes 2000) revealed that bulges can indeed form by dynamical evolution of disks through bars. If a bar extends about up to the corotation radius, a ring or tightly wound spiral structure can form. While the bar starts to decrease again in the further evolution, the spiral structure gets less tightly wound and a spherical bulge may evolve. Similar simulations have successfully described e.g. the bar/spiral structure of NGC 7331 (von Linden et al. 1996).

Bars can even form and dissolve several times during the evolution of a galaxy. Bournaud \& Combes (2002) concluded from their galaxy simulations that the pattern speed of the bar increases from one bar to the next while the new bar is shorter than the previous one. Hence a galaxy may be shifting progressively to early-types, with massive bulges. As the dissolving of a bar is a relatively short-living period compared to the galaxy's lifetime, we expect to observe this evolutionary phase only in a few number of galaxies.

We note here, that Emsellem \& Ferruit (2000) found strong indications for the existence of a nuclear bar in NGC 4594 inside a radius of $20^{\prime \prime}$ from the nucleus.

The view of NGC 4594 of a "normal" early type, spiral galaxy seen edge-on is further supported by the magnetic field structure that we deduced from our observations: the magnetic field orientation is mainly parallel to the galactic disk for low $z$-values but shows also significant vertical components for higher $z$-values (cf. Sect. 4.2.1). Such a configuration has been observed for many other (also late-type) galaxies seen edge-on, like e.g. NGC 4631 (Krause 2003), NGC 5775 (Tüllmann et al. 2000), and recently for NGC 891 (Krause et al., in preparation).

It is interesting to note that the region of strong polarized intensity (cf. Fig. 5 at $\lambda 6.2 \mathrm{~cm}$, the map with the highest linear resolution) is just within the range between the inner and the outer Lindblad resonances: ILR $=20^{\prime \prime}$ and OLR $=120^{\prime \prime}$ as estimated by Emsellem (1995; and Emsellem et al. 1996). The latter authors separated the rotation velocity in that of the disk and the bulge and found a very fast increasing rotation curve for the disk with velocities of $280 \mathrm{~km} \mathrm{~s}^{-1}$ at a radius of only $6^{\prime \prime}$. The velocity in the HI/CO ring/spiral is about $350 \mathrm{~km} \mathrm{~s}^{-1}$ (Bajaja et al. 1991), hence the rotation curve between the ILR and OLR is rather flat. The strong differential rotation in this radial range supports the action of a mean field dynamo (e.g. Ruzmaikin et al. 1988) that may be responsible for the observed uniform magnetic field in NGC 4594.

In Fig. 5 we see two clear maxima at a radius of only about $40^{\prime \prime}(1.7 \mathrm{kpc})$ from the nucleus in linear polarization at $\lambda 6.2 \mathrm{~cm}$. This might indicate the existence of another ring or a spiral arm structure nearby. It should be possible to reveal such a structure by a detailed analysis of the HST images of NGC 4594.

\subsection{Cold dust}

The dust distribution in NGC 4594 was studied by Emsellem (1995) on the basis of multi-color images. This study used multi-Gaussian expansion technique to determine via the optical depth the mass of the dust. From this modeling they came to the conclusion that the major part of the dust $(>85 \%)$ should be cold $\left(T_{\mathrm{d}}<20 \mathrm{~K}\right)$ and concentrated between $100^{\prime \prime}$ and 200" distance from the nucleus. This could however not directly be observed by Emsellem.

From the noise level of our 40" HPBW map (rms = $40 \mathrm{mJy} /$ beam) we estimated that any extended emission in the disk of NGC 4594 adds up to a flux density $S_{870 \mu} \leq 0.2 \mathrm{Jy}$. This is significantly smaller than corresponding values for other spiral galaxies like e.g. NGC $4631\left(S_{870 \mu}=3.8 \mathrm{Jy}\right.$, Dumke et al. 2004) or NGC $3628\left(S_{870 \mu}=1.9 \mathrm{Jy}\right.$, Dumke et al. 2003).

We have plotted the upper limit for the flux density at $\lambda 870 \mu \mathrm{m}$ together with the IRAS values for NGC 4594 at $\lambda \lambda$ 25, 60, and $100 \mu \mathrm{m}$ (Young et al. 1989) in Fig. 9. Also the IRAS flux density values of NGC 4594 are rather low 


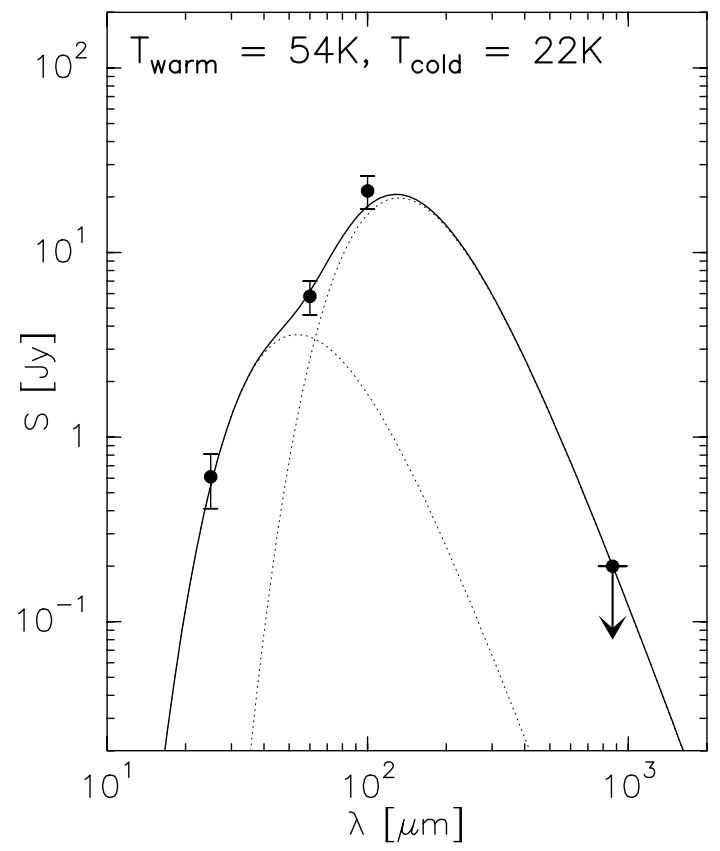

Fig. 9. The FIR-to-mm spectrum of NGC 4594. The FIR data points (IRAS) at $\lambda \lambda 25,60$, and $100 \mu \mathrm{m}$ are taken from Young et al. (1989), the $\lambda 870 \mu \mathrm{m}$ value is the upper limit for the disk emission from our HHT observations. The solid line shows a fit for a standard dust model (two modidied Planck curves with $\beta=2$ ).

compared to the corresponding values of the other 17 Sa galaxies in the sample of Young et al. (1989).

We fitted the FIR-to-mm spectrum with a dust model consisting of two components of large grains with different temperatures, both with $\beta=2$ (see below), similar to the procedure in Dumke et al. (2004) for NGC 4631. The result is also shown in Fig. 9 where the solid curve shows a two-component modified Planck spectrum with temperatures of $54 \mathrm{~K}$ and $22 \mathrm{~K}$. The location of the maximum of the cold component (and therefore its temperature) depends strongly on the data points at $\lambda 100 \mu \mathrm{m}$ and $\lambda 870 \mu \mathrm{m}$. Since the $\lambda 870 \mu \mathrm{m}$ value is an upper limit, we can only obtain a lower limit for the temperature of the cold component: because of Wien's law, decreasing $S_{870 \mu}$ would lead to a higher fitted dust temperature. Similarly, decreasing $S_{100 \mu}$ would lead to a lower fitted temperature. Thus - to really obtain a lower limit - we used $(S-\delta S)_{100 \mu \mathrm{m}}$ (i.e. the lower edge of the error bar) as $S_{870 \mu}$ data point for the temperature fit, as it can be seen in Fig. 9.

This lower limit for the temperature of the cold dust component enables us to roughly estimate an upper limit for the cold dust mass in NGC 4594. Following the procedure in Emsellem (1995), using an average grain size of $0.1 \mu \mathrm{m}$, the grain density and emissivity given by Hildebrand (1983) for $\lambda 870 \mu \mathrm{m}$ with $\beta=2$ and the relation

$M_{d}^{\mathrm{IRAS}}=1.2 \times 10^{-9} S_{\nu} D_{\mathrm{Mpc}}^{2} \lambda_{\mu}^{4}\left[\exp \left(\frac{1.44 \times 10^{4}}{\lambda_{\mu} T_{\mathrm{d}}}\right)-1\right]$

where $S_{v}$ is the IRAS flux in mJy at wavelength $\lambda(\mu \mathrm{m})$, distance $D(\mathrm{Mpc})$ and temperature $T_{\mathrm{d}}(\mathrm{K})$. With $T=22 \mathrm{~K}$, the upper limit for the $\lambda 870 \mu \mathrm{m}$ flux infers an upper limit for the cold dust mass of $1.2 \times 10^{7} M_{\odot}$. The mass of the "warm dust" as given by Emsellem (1995) and corrected for the distance of $8.9 \mathrm{Mpc}$ is $1.2 \times 10^{6} M_{\odot}$. Hence, our upper limit for the cold dust mass lies well above Emsellem's value for the warm dust and allows that more than $85 \%$ of the dust mass is indeed cold as inferred from Emsellem's light scattering model.

However, the observation of the cold $(T \simeq 20 \mathrm{~K})$ dust in the submm wavelength range is quite difficult because of the exponential dependence of the dust mass on the dust temperature and the observed wavelength (cf. the formula above). The expected flux density is much higher at wavelengths between $\lambda 100$ and $200 \mu \mathrm{m}$. Indeed, the detection of a dust ring in NGC 4594 at $\lambda 160 \mu \mathrm{m}$ by Spitzer observations has recently been reported by Bendo et al. (2004).

The total HI mass in NGC 4594 is estimated to be $3.0 \times$ $10^{8} M_{\odot}$ (Bajaja et al. 1984) and the total $\mathrm{H}_{2}$ mass is $4.4 \times$ $10^{8} M_{\odot}$ (Bajaja et al. 1991), both corrected for our adopted distance of 8.9 Mpc. Hence, the total gas mass in NGC 4594 is $7.4 \times 10^{8} M_{\odot}$. The estimated upper limit for the mass of the cold dust is $1.6 \%$ of the total gas mass. This upper value for NGC 4594 is equal to the value in the Milky Way (Sodroski et al. 1994) and at the lower end of the range estimated for M31 (Nieten et al. 2005).

\subsection{Star formation rates and efficiencies}

As mentioned above, the FIR to submm flux densities of NGC 4594 are rather weak compared to other galaxies. The FIR luminosity of NGC 4594 as given by Young et al. (1989) and corrected for the distance of $8.9 \mathrm{Mpc}$ is $L_{\mathrm{FIR}}=1.86 \times$ $10^{9} L_{\odot}\left(\right.$ respectively $\left.7.1 \times 10^{35} \mathrm{~W}\right)$, hence $\log \left(L_{\mathrm{FIR}} / L_{\odot}\right)=9.27$. The radio flux density of the disk of NGC 4594 at $\lambda 20 \mathrm{~cm}$ is $13.4 \mathrm{mJy}$ (Bajaja et al. 1988), giving a radio luminosity of $L_{1.49 \mathrm{GHz} \text {,disk }}=1.26 \times 10^{20} \mathrm{~W} \mathrm{~Hz}^{-1}$. The corresponding data point lies at the low radio luminosities of the radio-FIRcorrelation of Condon et al. (1991) and of Niklas (1997). In both samples of the radio-FIR relation the total radio luminosity has been considered, including the nuclear fluxes of the galaxies. If we include the nuclear flux also for NGC 4594, the total radio luminosity is $L_{1.49 \mathrm{GHz}}=1.08 \times 10^{21} \mathrm{~W} \mathrm{~Hz}^{-1}$. With this radio luminosity, NGC 4594 fits right above the best fit line in the radio-FIR correlation diagram of Niklas (1997).

The molecular gas as derived from CO measurements by Bajaja et al. (1991) (corrected for the distance of $8.9 \mathrm{Mpc}$ ) is $M_{\mathrm{H}_{2}}=4.4 \times 10^{8} M_{\odot}$. This leads to a star formation efficiency SFE of $4.2 L_{\odot} / M_{\odot}$ according to the definition of Young et al. (1989). The SFE for early-type galaxies does not generally differ from those of late-type galaxies (Thronson et al. 1989) and the value of NGC 4594 is rather but not extremely low.

The star formation rate SFR can be estimated from the $\mathrm{H}_{\alpha}$ or FIR luminosities which leads for NGC 4594 to quite different values. Following the procedure described by Thronson et al. (1989) we derive from the $\mathrm{H}_{\alpha}$ flux density of Schweizer (1978) a SFR of $\dot{M}_{\mathrm{H} \alpha}=0.07 M_{\odot} \mathrm{yr}^{-1}$ for $D=8.9 \mathrm{Mpc}$ which is only about 20-30\% of the SFR in M 31 (Walterbos 2000). From the FIR luminosity we derive with the formula given by Thronson et al. (1989) a SFR of $\dot{M}_{\mathrm{FIR}}=1.2 M_{\odot} \mathrm{yr}^{-1}$. The difference between $\dot{M}_{\mathrm{H} \alpha}$ and $\dot{M}_{\mathrm{FIR}}$ may partly be due to the dust 
that leads to extinction of the $\mathrm{H}_{\alpha}$ emission. Simultaneously, there may be additional FIR radiation by a cool "cirrus" component that does not come from star formation but from dust heated by the general interstellar radiation field. Furthermore, the FIR emission includes the nuclear emission as well. Its contribution in the (submm) range is more than $50 \%$ and may be similar in the FIR.

According to Niklas \& Beck (1997) the SFR scales with the equipartition magnetic field strength as $S F R \propto B^{2.92 \pm 0.70}$. The averaged equipartition magnetic field strength of a sample of 74 spiral galaxies is $9 \pm 3 \mu \mathrm{G}$ (Niklas 1995). While the total equipartition field strength for $|r| \simeq 20^{\prime \prime}$ to $110^{\prime \prime}$ is $6 \pm 1 \mu \mathrm{G}$, the averaged total magnetic field strength in NGC 4594 was estimated to be $4 \pm 1 \mu \mathrm{G}$ (Sect. 4.2.2). This value is below the one of Niklas' sample and somewhat lower than the averaged total field strength of M 31 (type $\mathrm{Sb}$ ) and M 33 (type Sc) (which is $6 \pm 2 \mu \mathrm{G}$ according to Beck 2000). The above relation expects the SFR in NGC 4594 to be a factor of about 10 lower than galaxies with an averaged total field strength of $9 \mu \mathrm{G}$. With the field strength given for M 31, the SFR in NGC 4594 is expected to be $30 \%$ of the value for M 31 in accordance with the SFRs for both galaxies as estimated from their $\mathrm{H}_{\alpha}$ emission (see last chapter).

\section{Summary}

We observed NGC 4594 in a wide wavelength range in radio continuum: with the VLA at $\lambda 6.2 \mathrm{~cm}$ in its D-array, with the Effelsberg $100-\mathrm{m}$ telescope at $\lambda 3.6 \mathrm{~cm}$, both also in linear polarization, and with the HHT at $\lambda 870 \mu \mathrm{m}$. At $\lambda 6.2 \mathrm{~cm}$ we detected extended disk emission with a similar distribution along the major axis as at $\lambda 20 \mathrm{~cm}$ by Bajaja et al. (1988) and some extended spurs suggesting outflows.

From the $\lambda 6.2 \mathrm{~cm}$ total intensity we also determined the thickness of the galactic disk in NGC 4594. Least-square fits to the $z$-distribution show lowest $\chi^{2}$ values for a single component Gaussian distribution different from other spiral galaxies seen edge-on whose $z$-distribution can be best described by a two-component exponential function (with scale heights of $300 \mathrm{pc}$ and $1.8 \mathrm{kpc}$ ) (Dumke \& Krause 1998). The resultant scale height for the disk thickness in NGC 4594 is $1.5 \mathrm{kpc}\left(35^{\prime \prime}\right)$ within $r= \pm 100^{\prime \prime}$ ( $4.3 \mathrm{kpc}$ ). The Gaussian shape may be due to the huge bulge in NGC 4594 as it is expected for a relatively thin layer (the disk) inside a nearly spherical potential (Combes 1991).

For the first time, we detected extended linear polarization in the radio range in NGC 4594 with an average degree of polarization of $15 \pm 3 \%$ over the whole galaxy and local values as high as $20 \%$ to $30 \%$. This is to our knowledge the first detection of a large-scale magnetic field in an Sa galaxy in the radio range.

The Faraday rotation could be determined between $\lambda 6.2 \mathrm{~cm}$ and $\lambda 3.6 \mathrm{~cm}$ to be in the range of $-200 \leq \mathrm{RM} \leq 200 \mathrm{rad} / \mathrm{m}^{2}$ with most values between -100 and $100 \mathrm{rad} / \mathrm{m}^{2}$. Correcting for Faraday rotation leads to the intrinsic magnetic field orientation which is parallel to the galactic disk in the midplane except in the innermost $50^{\prime \prime}$. There and generally at higher $z$-value above and below the disk the magnetic field has also significant vertical components.

Under the assumption of equipartition the magnetic field strength in NGC 4594 was estimated to be $6 \pm 1 \mu \mathrm{G}$ for $|r| \simeq$ $20^{\prime \prime}$ to $110^{\prime \prime}$, whereas the averaged total magnetic field strength in NGC 4594 was estimated to be $4 \pm 1 \mu \mathrm{G}$ (Sect. 4.2.2). Hence, the magnetic field strengths in NGC 4594 are in the lower range of those of normal spiral galaxies.

The polarized intensity is concentrated in the radial range between the ILR and OLR as estimated by Emsellem (1995) and Emsellem et al. (1996). In this radial range, the rotation velocity is as high as about $300 \mathrm{~km} \mathrm{~s}^{-1}$ and the rotation curve of the disk is only slightly increasing. The strong differential rotation there supports the action of a mean field dynamo that may be responsible for the observed uniform magnetic field in NGC 4594.

At $\lambda 870 \mu \mathrm{m}$ we detected the central source with a flux density of $S_{870 \mu \mathrm{m}}=230 \pm 35 \mathrm{mJy}$. We could not detect extended emission at this wavelength with our sensitivity of $40 \mathrm{mJy} /$ beam with 40" HPBW. However, we estimated an upper limit of a possible dust emission between 100" $\leq r \leq$ 200 arcsec (as expected e.g. by Emsellem (1995)) below our noise level to $S_{870 \mu} \leq 0.2 \mathrm{Jy}$.

A two-component fit of the dust spectrum (our upper limit for $S_{870 \mu}$ together with the IRAS values of Young et al. 1989) gives a lower limit for the temperature of the cold dust component of $T \leq 22 \mathrm{~K}$. This temperature enables us to roughly estimate an upper limit for the cold dust mass in NGC 4594 to $1.2 \times 10^{7} M_{\odot}$ which agrees with the cold dust mass claimed by Emsellem (1995) in his model including light scattering by dust.

Acknowledgements. We thank the staff of the HHT, the VLA and the Effelsberg 100-m telescope for their excellent support. We acknowledge discussions with S. v. Linden about galaxy evolution in simulations and thank R. Beck for helpful comments on the manuscript.

\section{References}

Baars, J. W. M., Genzel, R., Pauliny-Toth, I. I. K., \& Witzel, A. 1977, A\&A, 61, 99

Baars, J. W. M., Martin, R. N., Mangum, J. G., McMullin, J. P., \& Peters, W. L. 1999, PASP, 111, 627

Bajaja, E., van der Burg, G., Faber, S. M., et al. 1984, A\&A, 141, 309

Bajaja, E., Hummel, E., Wielebinski, R., \& Dettmar, R.-J. 1988, A\&A, 202, 35

Bajaja, E., Krause, M., Wielebinski, R., \& Dettmar, R.-J. 1991, A\&A, 241,411

Beck, R. 2000, in The interstellar medium in M31 and M33, ed. E. M. Berkhuijsen, R. Beck, \& R. A .M. Walterbos, WE-Heraeus Seminar, Shaker Verlag, Aachen, Proc. 232, 171

Beck, R. 2004, Ap\&SS, 289, 293

Beck, R., \& Krause, M. 2005, Astronomical Notes, 326, 414

Bendo, G. J., Armus, L., Calzetti, D., et al. 2004, American Astronomical Society Meeting Abstracts, 205, 60.06

Bournaud, F., \& Combes, F. 2002, A\&A, 392, 83

Burkhead, M. S. 1986, AJ, 91, 777

Christian, C. A., Bond, H. E., Frattare, L. M., et al. 2003, American Astronomical Society Meeting Abstracts, 203, 116.11

Combes, F. 1991, ARA\&A, 29, 195 
Combes, F. 2000, in Building Galaxies; from the Primordial Universe to the Present, 413

Condon, J. J., Anderson, M. L., \& Helou, G. 1991, ApJ, 376, 95

de Bruyn, A. G., Crane, P. C., Price, R. M., \& Carlson, J. B. 1976, A\&A, 46, 243

Dettmar, R. 1986, Ph.D. Thesis, University of Bonn

Dumke, M., \& Krause, M. 1998, in The Local Bubble and Beyond, ed. D. Breitschwerdt, M. Freyberg, \& J. Trümper (Berlin: SpringerVerlag), Proc. IAU Coll. 166, LNP Vol. 506, 555

Dumke, M., Krause, M., Wielebinski, R., \& Klein, U. 1995, A\&A, 302, 691

Dumke, M., Krause, M., \& Wielebinski, R. 2000, A\&A, 355, 512

Dumke, M., Krause, M., \& Wielebinski, R. 2003, in The Neutral ISM in Starburst Galaxies, ed. S. Aalto, S. Hüttemeister, \& A. Pedlar, ASP Conf. Ser., 320, 134

Dumke, M., Krause, M., \& Wielebinski, R. 2004, A\&A, 414, 475

Emerson, D. T., \& Graeve, R. 1988, A\&A, 190, 353

Emsellem, E. 1995, A\&A, 303, 673

Emsellem, E., \& Ferruit, P. 2000, A\&A, 357, 111

Emsellem, E., Bacon, R., Monnet, G., \& Poulain, P. 1996, A\&A, 312, 777

Ford, H. C., Hui, X., Ciardullo, R., Jacoby, G. H., \& Freeman, K. C. 1996, ApJ, 458, 455

Heckman, T. M. 1980, A\&A, 87, 152

Hildebrand, R. H. 1983, QJRAS, 24, 267

Kormendy, J. 1988, ApJ, 335, 40

Kormendy, J., Bender, R., Ajhar, E. A., et al. 1996, ApJ, 473, L91+

Krause, M. 2003, in Radio Studies of Galactic Objects, Galaxies, and AGNs, ed. J. L. Han, X. H. Sun, J. Yang, \& R. Wielebinski, Proc. Sino-German Radio Astronomy Conference, Acta Astron. Sinica, 44,123
Nieten, C., Neininger, N., Guélin, M., et al. 2005, A\&A, submitted Niklas, S. 1995, Ph.D. Thesis, University of Bonn

Niklas, S. 1997, A\&A, 322, 29

Niklas, S., \& Beck, R. 1997, A\&A, 320, 54

Niklas, S., Klein, U., \& Wielebinski, R. 1997, A\&A, 322, 19

Rubin, V. C., Burstein, D., Ford, W. K., \& Thonnard, N. 1985, ApJ, 289,81

Ruzmaikin, A. A., Sokolov, D. D., \& Shukurov, A. M. 1988, Magnetic fields of galaxies (Moscow, Izdatel'stvo Nauka), in Russian, 280

Scarrott, S. M., White, C., Pallister, W. S., \& Solinger, A. B. 1977, Nature, 265, 32

Schweizer, F. 1978, ApJ, 220, 98

Simard-Normandin, M., Kronberg, P. P., \& Button, S. 1981, ApJS, 45, 97

Sodroski, T. J., Bennett, C., Boggess, N., et al. 1994, ApJ, 428, 638

Sokoloff, D. D., Bykov, A. A., Shukurov, A., et al. 1998, MNRAS, 299, 189

Tüllmann, R., Dettmar, R.-J., Soida, M., Urbanik, M., \& Rossa, J. 2000, A\&A, 364, L36

Thronson, H. A., Tacconi, L., Kenney, J., et al. 1989, ApJ, 344, 747

van der Burg, G., \& Shane, W. W. 1986, A\&A, 168, 49

von Linden, S., Reuter, H.-P., Heidt, J., Wielebinski, R., \& Pohl, M. 1996, A\&A, 315, 52

Wagner, S. J., Bender, R., \& Dettmar, R.-J. 1989, A\&A, 215, 243

Wainscoat, R. J., Hyland, A. R., \& Freeman, K. C. 1990, ApJ, 348, 85

Walterbos, R. A. M. 2000, in The interstellar medium in M 31 and M 33, ed. E. M. Berkhuijsen, R. Beck, \& R. A. M. Walterbos, WE-Heraeus Seminar, Shaker Verlag, Aachen, Proc. 232, 99

Young, J. S., Xie, S., Kenney, J. D. P., \& Rice, W. L. 1989, ApJS, 70, 699 